\title{
Comparative effectiveness of correction of cervical incompetence through cerclage and obstetric pessary
}

Khamoshina M.B., MD, PhD, Professor of Department

Zamotina I.V., post-graduate student of Department

Radzinsky V.E., MD, PhD, Professor of Department, head of Department

Lebedeva M.G., PhD, associate professor of Department

Kostin I.N., MD, PhD, Professor of Department

Russia, Moscow, People's Friendship University of Russia, Department of Obstetrics and Gynecology

\section{Broblem statement}

Preterm birth is a global problem of obstetrics and perinatology. Complications resulting from premature births are the main cause of death of children under five years of age (Information Note of the WHO, 2016). The consequences of cervical incompetence (CI) are up to $40 \%$ of prematurely completed gestations.

Objective of the study: improvement of the outcome of pregnancy and childbirth in the patients with CI.

Methods: the study was performed in 2012-2015 in the clinical bases of PFUR. Pregnant women with CI were examined and treated at 18-22 week $(n=221)$. The CI criterion was considered to be a vaginal ultrasound data: cervix lengh $(\mathrm{CL}) \leq 25 \mathrm{~mm}$. The patients were randomized to groups: I - cerclage correction (circular mersilene tape) $(n=107)$; II - pessary correction ("Juno" manufactured by Simurg, Belarus) $(n=114)$. The main parameters of the subjects of both groups were comparable. The effectiveness of the correction was determined in 2-3 weeks after putting of the pessary or suturing of the cervix uteri according to the ultrasound data, the effectiveness criterion was considered to be the length of the CL> $25 \mathrm{~mm}$, and the width of the cervical canal<10 mm. To determine the validity of the differences, we used the Student's test under normal distribution, and the Mann-Whitney test (UTest) as the nonparametric method. The methods of variance statistics (correlative, multi-factor, variance analysis, logistic regression analysis) and the probability relationship calculation were used. The differences were considered to be statistically credible at $\mathrm{p}<0.05$.

\section{Results}

The analysis of the gestation course showed that the main complication of the gestation after the correction was an imminent abortion (O0.20). This complication was determined in $194(87.8 \%)$ patients, in the cerclage group - 94.3\%, in the pessary group $-81.5 \%(\mathrm{p}<0.05)$. In I group one pregnant woman had $2.2 \pm 0.6$ hospital admissions, while in II $-1.3 \pm 0.8$, i.e. after correction with the pessary, the women were hospitalized 1.7 times less frequently $(\mathrm{p}<0.05)$. In general, the effectiveness of the CIC correction was $90.5 \%$, in I - 91.6\%, in II $-89.5 \%$. We determined the dependence of efficacy of the methods on $\mathrm{pH}$ of the vaginal medium, CL and history of preterm birth. If $\mathrm{pH}$ of the vagina was 4.0-5.3, the efficiency of the pessaries was $92.1 \%$, cerclage $-94.8 \%$, if $\mathrm{pH}>5.3$, the efficiency of the cerclage was reduced to $72.3 \%$, but it remained high enough in the pessary group (85.2\%). At $\mathrm{CL}<15$ $\mathrm{mm}$, the cerclage proved to be more efficient $(86.4 \%, 73.8 \%$ respectively) $(\mathrm{p}<0.05)$. In the patients with preterm births in past medical history, the correction of the CI was ineffective in I group - 3.4 times more frequently, in II - $3.0(\mathrm{p}<0.05)$.

In the analysis of the births, it was found that $85.0 \%$ of the patients with $\mathrm{CI}$, who had been cerclaged, and $77.1 \%$ of the patinets with CI who had been put the pessary, delivered in due time. The premature births were in $13.1 \%$ and $17,5 \%$ respectively. Spontaneous miscarriages occurred more frequently in the II group (5.3\% vs. $1.9 \%$ ).

\section{Conclusions}

The choice of the CI correction method on an individual basis depending on the cervical lengh, the $\mathrm{pH}$ of the vagina, the presence of premature births in a past medical history will allow prolonging the pregnancy with $\mathrm{CI}$, reducing the number of premature births. 